\title{
THE FAST MULTIPOLE METHOD FOR THE DIRECT E/MEG PROBLEM
}

\author{
Maureen Clerc ${ }^{1,2}$, Olivier Faugeras ${ }^{2}$, Renaud Keriven $^{1}$, Jan Kybic ${ }^{2}$, Théo Papadopoulo ${ }^{2}$ \\ ${ }^{1}$ Odyssée project \\ CERMICS \\ ${ }^{2}$ Odyssée Project \\ INRIA \\ Ecole Nationale des Ponts et Chaussées \\ 2004 Route des Lucioles, BP93 \\ 77455 Marne-La-Vallée Cedex 2 \\ France \\ 06902 Sophia-Antipolis \\ France
}

\begin{abstract}
Reconstructing neuronal activity from MEG and EEG measurements requires the accurate calculation of the electromagnetic field inside the head. The boundary element formulation of this problem leads to a dense linear system which is too large to be solved directly. We propose to accelerate the computations via the fast multipole method. This method approximates the electromagnetic interaction between surface elements by performing multipole expansions at a coarse resolution. It significantly reduces the computational complexity of the matrix-vector products needed for the iterative solution of the linear system, and avoids the storage of its matrix. We describe the single-level fast multipole method and present several experiments demonstrating its accuracy and performance.
\end{abstract}

\section{INTRODUCTION}

Electro/magnetoencephalography (E/MEG) measures the small electric and magnetic fields on the surface of the skull [1]. The ultimate goal is to solve the inverse problem: reconstruct from these measurements the primary currents inside the cortex, due to brain activity. Its essential part is the solution of the forward problem: find the field caused by a known distribution of current sources. In this short article we focus on the computation of the electric potential since the magnetic field follows from the Biot-Savart law [1]. Anatomical data (MRI) can provide a head model [2] consisting of several regions of constant conductivity, representing for example the white matter, the cortex, the cerebrospinal fluid, the skull, and the scalp [3]. In the surface approach, the potential, sought at the boundaries between different regions, is governed by an integral equation, which can be solved numerically with the boundary element method (BEM).

Recent experiments indicate that existing BEM implementations suffer from unacceptably large errors when the current source approaches the volume discontinuity [4].
Unfortunately, this is precisely the case in the brain, where the main primary current sources are supposedly the pyramidal cells in the cortex, a layer only a few millimeters thick. Although the BEM is able to deal with complex surface geometry, realistic head models do not appear to offer greater accuracy than a simple concentric spheres model [5].

We believe that one of the reasons for this inadequacy is the simplicity of the surface model employed: the boundaries are represented with at most several thousand triangles that cannot capture the fine cortex geometry nor the important spatial variations of the potential. Unfortunately, the computational complexity and storage requirements of the direct calculation of pairwise interactions between elements quickly become prohibitive. Iterative techniques [6] avoid the storage at the expense of increased number of operations.

The fast multipole method (FMM) is a standard tool in particle simulations and computational electromagnetics. It reduces the asymptotical complexity of the interaction evaluation from $O\left(P^{2}\right)$, where $P$ is the number of parameters (degrees of freedom) in our model, ultimately down to $O(P \log P)$, which allows to use models with an order of several magnitudes more elements than with the classical approach.

\section{BOUNDARY ELEMENT METHOD}

\subsection{Surface equation}

The potential $V(\mathbf{r})$ on a smooth surface $S_{m}$ separating two regions with conductivities $\sigma_{m}^{+}, \sigma_{m}^{-}$satisfies the following integral equation $[4,7]$ : for $\mathrm{r} \in S_{m}$,

$$
\begin{aligned}
& \sigma_{0} V_{0}(\mathbf{r})=\frac{\sigma_{m}^{+}+\sigma_{m}^{-}}{2} V(\mathbf{r})+ \\
& \sum_{k=1}^{N} \frac{\sigma_{k}^{+}-\sigma_{k}^{-}}{4 \pi} \int_{\mathbf{r}^{\prime} \in S_{k}} V\left(\mathbf{r}^{\prime}\right) \nabla^{\prime}\left(\frac{1}{R}\right) \cdot \mathbf{n}_{k}\left(\mathbf{r}^{\prime}\right) \mathrm{d} s_{k}\left(\mathbf{r}^{\prime}\right)
\end{aligned}
$$


with $\mathbf{R}=\mathbf{r}-\mathbf{r}^{\prime}, R=\|\mathbf{R}\|$ and $\nabla^{\prime}\left(\frac{1}{R}\right)=\frac{\mathbf{R}}{R^{3}}$, where $N$ is the number of surfaces, $\mathrm{n}_{k}$ is the unit vector normal to $S_{k}$ oriented from the region with $\sigma_{k}^{-}$to the one with $\sigma_{k}^{+} . V_{0}$ is a potential due to a known primary current distribution $\mathbf{J}^{p}(\mathbf{r})$ in the infinite homogeneous space with conductivity $\sigma_{0}[7]$

$$
\sigma_{0} V_{0}(\mathbf{r})=\frac{1}{4 \pi} \int_{\mathbf{r}^{\prime} \in \mathbf{R}^{3}} \mathbf{J}^{p}\left(\mathbf{r}^{\prime}\right) \cdot \nabla^{\prime}\left(\frac{1}{R}\right) \mathrm{d} \mathbf{r}^{\prime}
$$

\subsection{Discretization}

Each surface $S_{k}$ is typically represented by a triangulation, a triangle is called a boundary element. We discretize the potential $V$ and the primary current $\mathbf{J}^{p}$

$$
\begin{aligned}
V(\mathbf{r}) & =\sum_{k=1}^{N} \sum_{i=1}^{P_{k}} v_{i k} \varphi_{i k}(\mathbf{r}) \\
\mathbf{J}^{p}(\mathbf{r}) & =\sum_{l=1}^{P_{J}} u_{l} \mathbf{n}_{l J} \phi_{l}(\mathbf{r})
\end{aligned}
$$

Each basis function $\varphi_{i k}, k=1, \ldots N, i=1 \ldots P_{k}$ is nonzero on only one element of $S_{k}$. Similarly, each $\phi_{l}$ is nonzero on only one element of the cortex surface $S_{J}$. There is one basis function per triangle for the piecewise-constant (P0) approximation, one per vertex for the piecewise-linear case (P1). We restrict $\mathrm{J}^{p}$ to be normal to $S_{J}$, which is physiologically motivated, and $\mathbf{n}_{l J}$ is the unit vector normal to the $l$ th element of $S_{J}$.

Variational formulations of (1) and (2) are obtained by taking the inner product between both sides of the equations and test-functions $\psi_{j m}$, which are nonzero only on $S_{m}$. Because of $(1), b_{j m}=\left\langle\sigma_{0} V_{0}, \psi_{j m}\right\rangle$ satisfies

$$
\begin{gathered}
b_{j m}=\frac{\sigma_{m}^{+}+\sigma_{m_{m}}^{-}}{2} \sum_{i=1}^{P_{m}} v_{i m}\left\langle\varphi_{i m}, \psi_{j m}\right\rangle \\
\quad+\sum_{k=1}^{N} \frac{\sigma_{k}^{+}-\sigma_{k}^{-}}{4 \pi} \sum_{i=1}^{P_{k}} v_{i k} \Gamma_{i k, j m}^{\mathbf{n}_{i k} \varphi_{i k}, \psi_{j m}} \\
\Gamma_{i k, j m}^{\mathbf{n}_{i k} \varphi_{i k}, \psi_{j m}}=\iint_{\substack{\mathbf{r} \in \operatorname{supp} \psi_{j m} \\
\mathbf{r}^{\prime} \in \operatorname{supp} \varphi_{i k}}} \nabla^{\prime}\left(\frac{1}{R}\right) \cdot \mathbf{n}_{i k} \varphi_{i k}\left(\mathbf{r}^{\prime}\right) \psi_{j m}(\mathbf{r}) \mathrm{d} s^{2}\left(\mathbf{r}^{\prime}, \mathbf{r}\right)
\end{gathered}
$$

which represents a linear system $b_{j m}=\sum_{i k} a_{i k, j m} v_{i k}$. For some choices of $\varphi ; \psi$, the integral $\Gamma$ can be calculated analytically [8]. Because of (2) and (4),

$$
b_{j m}=\frac{1}{4 \pi} \sum_{l=1}^{P J} u_{l} \Gamma_{l, j m}^{\mathbf{n}_{l} \phi_{l}, \psi_{j m}}
$$

which represents another linear system $b_{j m}=\sum_{l} u_{l} c_{i, j m}$. We therefore have $A v=b=C u$, where both $A$ and $C$
Table 1: For a current dipole at a distance $d$ from a unitary spherical surface, discretized with triangles with max edge-length $h \approx 0.3$, we show the relative error for the collocation methods $A: \varphi=\delta, \psi=\delta$ and $B: \varphi=P_{0}, \psi=\delta$; a semi-Galerkin method $C$; and full Galerkin methods $D: \varphi=P_{0}, \psi=P_{0}, 3$ point Gauss quadrature; and $E$ : as $\mathrm{D}$, but 10 point Gauss quadrature.

\begin{tabular}{|c||c|c|c|c|c|}
\hline $\mathrm{d}$ & $\mathrm{A}$ & $\mathrm{B}$ & $\mathrm{C}$ & $\mathrm{D}$ & $\mathrm{E}$ \\
\hline \hline 0.5 & 0.0182 & 0.0122 & 0.0103 & 0.0079 & 0.0079 \\
\hline 0.3 & 0.0243 & 0.0165 & 0.0167 & 0.0158 & 0.0158 \\
\hline 0.1 & 0.0565 & 0.0551 & 0.0640 & 0.0737 & 0.0734 \\
\hline 0.05 & 0.1899 & 0.1992 & 0.1173 & 0.1314 & 0.1358 \\
\hline 0.02 & 0.3524 & 0.3588 & 0.3614 & 0.3675 & 0.3484 \\
\hline 0.01 & 0.6414 & 0.6510 & 0.5235 & 0.5291 & 0.5137 \\
\hline 0.005 & 0.8739 & 0.8797 & 0.7269 & 0.7641 & 0.7231 \\
\hline
\end{tabular}

are weighted concatenations of matrices $\Gamma_{k, m}$. The direct $\mathrm{E} / \mathrm{MEG}$ problem consists of computing $\mathbf{v}$ from $\mathbf{u}$.

\subsection{Accuracy}

The accuracy of the BEM method varies with each specific implementation choice. However, no method can perform well unless the distance between the current source and the discontinuity is at least comparable to the typical element size, otherwise there are not enough degrees of freedom to describe the variations in the potential. Table 1 shows the relative $L_{2}$ error of the BEM-calculated potential as compared to an analytical solution [9]. We observe that approaching the source to the surface increases the error which becomes unacceptably large when the distance to the surface is less than about $h$. This coincides with other recent findings $[10,11]$.

Consequently, to accurately model the effects of current sources inside the cortex, a triangulation with an element size around $1 \mathrm{~mm}$ appears to be necessary. Given a cortex surface area of approximately $2500 \mathrm{~cm}^{2}$, we would ideally need about $10^{5} \sim 10^{6}$ triangles to represent the cortex adequately. Hence the interest in the FMM, which allows to process large numbers of triangles efficiently.

\section{FAST MULTIPOLE METHOD}

The FMM allows the computation of matrix-vector products $\Gamma x$ with a divide-and-conquer procedure. It does not require the explicit storage of the matrix coefficients, and its computational cost is only $O(P \log P)$ for a matrix $\Gamma$ of size $P \times P$. The FMM is an approximate method whose error can however be controlled. The main idea is to approximate the function of $r, r^{\prime}$ in the integral (6) by a sum of separable functions. In this article, we concentrate on a single-level FMM (sFMM), which is easy to describe. The transition to full, multi-level FMM is straightforward, albeit involved. 
The computational bulk of the matrix-vector products resides in the computation of expressions of the form $y_{j}=\sum_{i} \Gamma_{i, j}^{\mathbf{f}_{i}, g_{j}} x_{i}$ (see (6)), where $\mathbf{f}_{i}$ is nonzero inside an element $T_{i}$ with center $c_{i}$, and $g_{j}$ is nonzero inside an element $T_{j}$ with center $c_{j}$. The three-dimensional space is decomposed into a set of $C$ identically sized disjoint cubic cells $\mathcal{C}_{p}$ with centers $\mathbf{M}_{p}$ and neighborhoods (containing 26 cells each) $\mathcal{N}\left(\mathcal{C}_{p}\right)$.

Let $I_{n}^{m}(\mathbf{r})$ and $O_{n}^{m}(\mathbf{r})$ denote the inner and outer spherical harmonics [12]. We precalculate for each cell $\mathcal{C}_{p}$

$$
\begin{aligned}
\mathrm{a}_{n}^{-m}\left(\mathcal{C}_{p}\right) & =(-1)^{n} \sum_{\mathcal{C}_{i} \in \mathcal{C}_{p}} v_{i} \int_{\mathbf{r}^{\prime} \in T_{i}} \nabla^{\prime} I_{n}^{-m}\left(\mathbf{M}_{p}-\mathbf{r}^{\prime}\right) \mathbf{f}_{i}\left(\mathbf{r}^{\prime}\right) \mathrm{d} s\left(\mathbf{r}^{\prime}\right) \\
\mathrm{d}_{n^{\prime}}^{-m^{\prime}}\left(\mathcal{C}_{p}\right) & =\sum_{\substack{\mathcal{C}_{q} \notin \mathcal{N}\left(\mathcal{C}_{p}\right) \\
n=0 \ldots \infty \\
m=-n \ldots n}} O_{n+n^{\prime}}^{m+m^{\prime}}\left(\mathbf{M}_{p}-\mathbf{M}_{q}\right) \mathbf{a}_{n}^{-m}\left(\mathcal{C}_{q}\right)
\end{aligned}
$$

and for each element $T_{j} \in \mathcal{C}_{p}$

$$
\mathbf{b}_{n}^{-m}\left(T_{j}\right)=\int_{\mathbf{r} \in T_{j}} I_{n}^{-m}\left(\mathbf{M}_{p}-\mathbf{r}\right) g_{j}(\mathbf{r}) \mathrm{d} s(\mathbf{r})
$$

It is proved in [13] that, if $c_{j} \in \mathcal{C}_{q}$,

$$
y_{j}=\sum_{\substack{n^{\prime}=0 \ldots \infty \\ m^{\prime}=-n^{\prime} \ldots n^{\prime}}} \mathrm{d}_{n^{\prime}}^{-m^{\prime}}\left(\mathcal{C}_{q}\right) \mathbf{b}_{n^{\prime}}^{-m^{\prime}}\left(T_{j}\right)+\sum_{\substack{\left.\mathcal{C}_{p} \in \mathcal{N}\left(\mathcal{C}_{Q}\right) \\ c_{i} \in \mathcal{C}_{p}\right)}} x_{i} \Gamma_{i, j}^{\mathbf{f}_{i}, g_{j}}
$$

The infinite series over $n, n^{\prime}$ are in practice replaced by finite sums up to some a priori chosen truncation order $L$ : this explains why the FMM is an approximate method.

In summary, computing $y_{j}$ via the SFMM involves four steps corresponding to the four equations above: (I) A local computation of a inside each cell, precomputable for a given cortex geometry and discretization. (2) A coarselevel computation of $\mathrm{d}$ involving pairs of non-neighboring cells; $O\left(\mathbf{M}_{p}-\mathbf{M}_{q}\right)$ can be precomputed. (3) Another local computation of $b$ for all elements inside each cell. (4) A small number of interactions between elements from neighboring cells to be computed explicitly, possibly analytically.

Assuming uniformly distributed elements in space, the asymptotical computational complexity for the evaluation of $\Gamma \mathrm{x}$ by the sFMM is $O\left(L^{2} P+C^{2}+C(P / C)^{2}\right)$, which is minimized for $C_{\text {opt }} \sim P^{2 / 3}$, yielding a total computational cost of $O\left(P^{4 / 3}\right)$ (instead of $P^{2}$ for the direct method), with approximately $P^{1 / 3}$ elements per cell.

\section{NUMERICAL EXPERIMENTS}

The first experiment evaluates the accuracy of the sFMM method with respect to the direct method as a function of the truncation order $L$. We generated

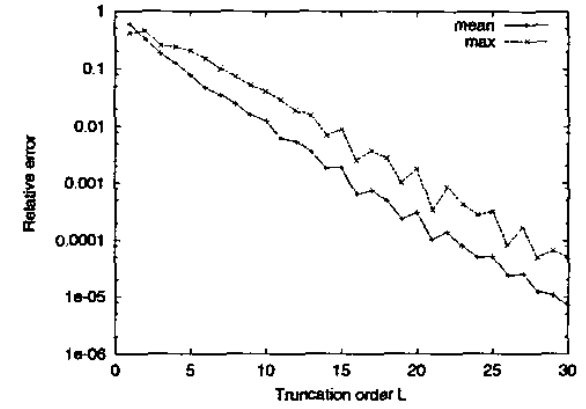

Fig. 1: Mean relative $\ell_{2}$ error and maximum relative error with respect to truncation order $L$.

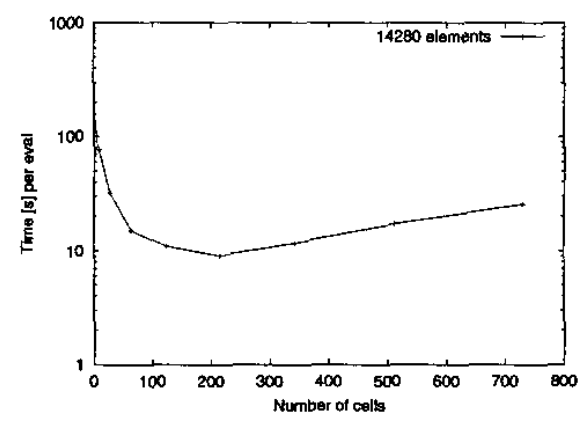

Fig. 2: Mean evaluation time with respect to the number of cells.

a small triangulated spherical surface with 56 triangles and compared the products $\Gamma \mathrm{e}_{i}\left(\mathbf{e}_{i}\right.$ are natural basis vectors) to their sFMM approximations $\tilde{\mathbf{\Gamma}} \mathbf{e}_{i}$. Fig. 1 shows the dependence of the mean relative $\ell_{2}$ error (typical case) $e_{2}^{\text {rel }}=\operatorname{mean}_{i}\left\|\Gamma \mathbf{e}_{i}-\tilde{\Gamma} e_{i}\right\|_{2} /\left\|\Gamma e_{i}\right\|_{2}$ and the maximum relative $\ell_{\infty}$ error (the worst case) $e_{\infty}^{\text {rel }}=\max _{i}\left\|\Gamma e_{i}-\tilde{\Gamma} e_{i}\right\|_{\infty} /\left\|\Gamma e_{i}\right\|_{\infty}$. We see that both

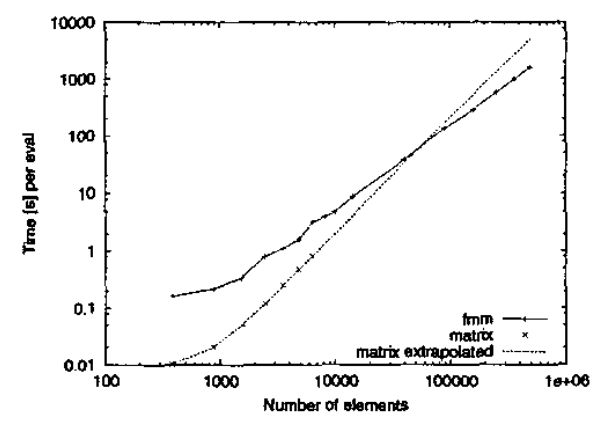

Fig. 3: Mean evaluation time for the sFMM and direct methods (extrapolated) with respect to the number of elements $P$. 


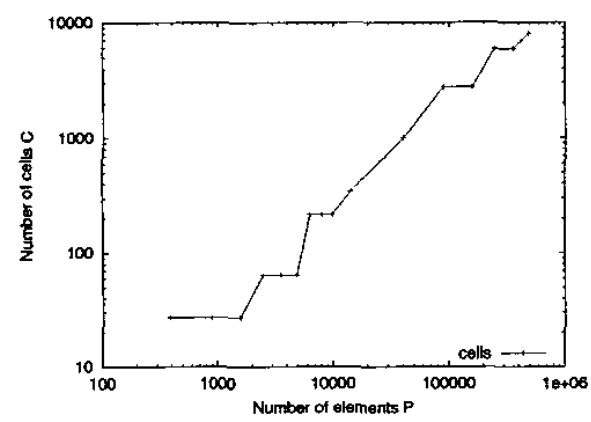

Fig. 4: The experimentally found optimum number of cells $C_{\mathrm{opt}}$ as a function of the number of elements $P$.

errors decrease steadily with the approximation order $L$. However, depending on the accuracy required, we might need to use relatively high orders $L$, in this case $L=15 \sim 20$.

Fig. 2 shows the mean time required to evaluate $\mathbf{\Gamma x}$ depending on the choice of the number of cells $C$, for a fixed number of elements $P=14280$ and a fixed order $L=5$. We observe that there is a geometry-dependent optimal number of cells $C_{\mathrm{opt}}$, somewhat different from the theoretical value derived in the preceding section for the uniform case. For our spherical triangulations, the optimum $C_{\text {opt }}$ is typically situated between $P^{1 / 2}$ and $P^{2 / 3}$ (c.f. Fig. 4).

Finally the third experiment (Fig. 3) compares the computational complexity of the SFMM algorithm with the direct method for progressively increasing number of elements $P$, for $L=5$, and with an experimentally found optimal $C$ (shown in Fig. 4). We observe that above $P \approx$ 70000 , it pays off to use the sFMM method, the full FMM will likely shift the crossing point even lower. The times for the direct method were quadratically extrapolated for high $P$, where it failed for the lack of memory. Note that, if the coefficients $\Gamma_{i, j}$ in the direct method were not stored but instead calculated as needed, the FMM would become even more competitive.

\section{CONCLUSIONS}

We have presented a new FMM (in its simplified sFMM form) for the efficient evaluation of matrix-vector products encountered in the BEM formulation of the direct E/MEG problem. This will permit the use of significantly larger and more precise head models, improving the accuracy of the solution of the direct - and consequently also inverse E/MEG problem. We have shown that the method is viable and outperforms the classical approach for fine triangulations. Future extensions to full FMM are likely to improve these results even further.

\section{ACKNOWLEDGMENTS}

The authors thank G. Sylvand for his advice on the FMM.

\section{REFERENCES}

[1] Matti Hämäläinen, Rïtta Hari, Risto J. Ilmoniemi, Jukka Knuutila, and Olli V. Lounasmaa, "Magnetoencephalography - theory, instrumentation, and applications to noninvasive studies of the working human brain," Reviews of Modern Physics, vol. 65, no. 2, pp. 413-497, Apr. 1993.

[2] Jyrki Lötjönen, Construction of Boundary Element Models in Bioelectromagnetism, Ph.D. thesis, Helsinki University of Technology, Espoo, Finland, 2000.

[3] Carlos H. Muravchik and Arye Nehorai, "EEG/MEG error bounds for a static dipole source with a realistic head model," IEEE Trans. Sig. Proc., vol. 49, no. 3, pp. 470-476, Mar. 2001.

[4] John C. Mosher, Richard B. Leahy, and Paul S. Lewis, "EEG and MEG: Forward solutions for inverse methods," IEEE Transactions on Biomedical Engineering, vol. 46, no. 3, pp. 245-259, Mar. 1999.

[5] R.M. Leahy, J.C. Mosher, M.E. Spencer, M.X. Huang, and J.D. Lewine, "A study of dipole localization accuracy for MEG and EEG using a human skull phantom," Los Alamos Technical Report LA-UR-98-1442, Los Alamos National Laboratory, Mar. 1998, Revision of LA-UR-97-4804.

[6] Jussi Rahola and Satu Tissari, "Iterative solution of dense linear systems arising from boundary element formulations of the biomagnetic inverse problem," Tech. Rep. TR/PA/98/40, CERFACS, 1998, Toulouse, France.

[7] O. Faugeras, F. Clément, R. Deriche, R. Keriven, T. Papadopoulo, J. Roberts, T. Viéville, F. Devernay, J. Gomes, G. Hermosillo, P. Kornprobst, and D. Lingrand, "The inverse EEG and MEG problems: The adjoint space approach I: The continuous case," Tech. Rep. 3673, INRIA, May 1999.

[8] J. C. de Munck, "A linear discretization of the volume conductor boundary integral equation using analytically integrated elements," IEEE Trans. Biomed. Eng., vol. 39, no. 9, pp. 986-990, Sept. 1992.

[9] Zhi Zhang, "A fast method to compute surface potentials generated by dipoles within multilayer anisotropic spheres," Phys. Med. Biol., vol. 40, pp. 335-349, 1995.

[10] Satu Tissari and Jussi Rahola, "Error analysis of a new Galerkin method to solve the forward problem in MEG and EEG using the boundary element method," Tech. Rep. TR/PA/98/39, CERFACS, 1998, Toulouse, France.

[11] A. Steward Ferguson and Gerhard Stroink, "Factors affecting the accuracy of the boundary element method in the forward problem - I: Calculating surface potentials," IEEE Trans. Biomed. Eng., vol. 44, no. 11, pp. 1139-1155, Nov. 1997.

[12] M.A. Epton and B. Dembart, "Theory for the threedimensional Laplace and Helmholtz equations," SIAM J. SCi. Comput., vol. 4, no. 16, pp. 865-897, 1995.

[13] Maureen Clerc, Renaud Keriven, Olivier Faugeras, Jan Kybic, and Théo Papadopoulo, "The BEM for EEG and MEC problems using FMM," Tech. Rep., INRIA, 2002, to appear. 